\title{
EDITORIAL
}

\section{Caribe más que moderno-occidental}

\author{
William Andrés Martínez Dueñas ${ }^{1}$
}

"The Caribbean is nothing but contact" Trouillot, (1992:22).

"La modernidad crea a sus otros: múltiples, multifacéticos, multidimensionales.

Lo ha hecho desde el primer día: nosotros siempre hemos sido modernos, modernos diferentes, contradictoriamente modernos, modernos de otro modo, pero modernos, sin duda"

Trouillot (2011, p. 94)

\begin{abstract}
Es difícil definir si la categoría Caribe, en su enfoque explícitamente político, pretende convocar - las fuerzas de un heterogéneo grupo de comunidades imaginadas o si por el contrario proyecta homogeneizar, neutralizar y capturar la multiplicidad de identidades cambiantes e híbridas que comparten el Mar Caribe y las cuencas continentales que a él llegan. La estrategia geopolítica se articula con el (los) proceso(s) de construcción de identidad; estos procesos de identificación Caribe no escapan a la dialógica representacional nosotros/otros ${ }^{2}$, cuya expresión más popular es la dicotomía Occidente/El Resto. Para el caso Caribe, estas dicotomías también son múltiples, por ejemplo para el caso colombiano se ha construido, quizá recientemente, la dicotomía Caribe/Andes que implica una constante discusión sobre dónde comienza y termina la una y la otra y quién es y no es Caribe, donde las dos representaciones son igualmente monolíticas y reduccionistas; pero si miramos fuera de Colombia encontramos la pareja Caribe/Atlántico Norte, donde Caribe puede ser entendido como una construcción de la imaginación de individuos del Atlántico Norte, que se naturalizó y produjo una modernidad alternativa aún por etnografíar y entender (Trouillot, 2011); así mismo en algunas versiones el Caribe se construye como el lugar idílico del eterno verano litoral (cf. Guerrón-Montero, 2011) en contraposición al paisaje urbano industrial. Por otro lado, en la ficción el Caribe es visto como el lugar de la aventura, habitado por salvajes y excluidos, en contraste con los espacios civilizados de los imperios del siglo XVI.
\end{abstract}

1. Editor en jefe revista Jangwa Pana - Dr. Antropología. Profesor asociado programa de Antropología, Facultad de Humanidades, Universidad del Magdalena. Colombia. Correo electrónico: wmartinez@unimagdalena.edu.co. ORCID ID: 0000-0003-0921-1149

2. Sobre la dialógica representacional ver Martinez-Dueñas y Perafán (2018). 
Este espacio geopolítico, llámese Gran Caribe, Cuenca del Caribe o Caribe a secas, no solo implica varias lenguas (y proyectos) imperiales (español, francés e inglés), sino un número mayor de lenguas prehispánicas que aún perviven y que entre otras quedan excluidas de los relatos que se perpetúan en medios de divulgación como éste, cuyo nombre, Jangwa Pana, es evidencia de la hipocresía multicultural (cargada de buenas intenciones ${ }^{3}$ ). Así mismo este gran territorio incluye un número increíble de grupos humanos, de territorialidades y por qué no decirlo de ontologías, para usar un término que ayuda a evadir la trampa multicultural ${ }^{4}$ y a pensar ecologías que incluyen seres como Bondy $^{5}$ o Serankua ${ }^{6}$. Y por si esto fuera poco el Caribe, está en medio de un flujo constante de mercancías e ideologías desde el siglo XVI en el sistema mundo moderno colonial $^{7}$, lo cual lo insertó tempranamente al sistema global de producción de capital y lo ha excluido de los privilegios de bienestar que éste ha producido, no sin antes dejar implantados los mismos deseos y anhelos de sus vecinos, los habitantes del atlántico norte ${ }^{8}$. Además, actualmente el conjunto de islas y litorales continentales que se vinculan al Caribe incluyen enclaves turísticos para las élites del Norte geopolítico, paraísos fiscales, circuitos transnacionales de narcotráfico y maquilas de

\footnotetext{
3. Sobre el nombre de la revista ver la editorial del Vol. 16, No. 1 (Martínez-Dueñas y Arias-Ocampo, 2017).

4. Para una discusión sobre esta trampa ver Martínez-Dueñas y Perafán (2017) y Martinez-Dueñas (2016).

5. En el Vudú Haitiano es una entidad superior, ver por ejemplo Jenson (2010).

6. Ser que habita el mundo de las comunidades indígenas de la Sierra Nevada de Santa Marta, territorio que algunos incluyen en la geografía múltiple del Caribe, ver Balanta (2015).

7. La idea de sistema mundo moderno colonial es la unión de las propuestas de Wallasrtein y los teóricos de la Modernidad/colonilidad como Mignolo.

8. Ver artículo de Camacho-Navarro (2018) en este número sobre los imaginarios del progreso en el Caribe. Así mismo ver el artículo de Robles-Santana (2018) en este número sobre el proceso de migración de mujeres Mam guatemaltecas a fincas cafeteras en México. El artículo de Herrera-Llamas, Alvarado-De Lima y Herrera-Aguilar (2018), también en este número ofrece elementos teóricos para entender las migraciones regionales y la desigualdad social que estas perpetúan en vez de mitigar.
}

corporaciones transnacionales (cf. Mackenbach, 2011), solo por mencionar algunas cosas que se conectan en este espacio.

Estos procesos implican no solo el intercambio de bienes, servicios e ideologías, sino también el intercambio de individuos y sociedades, que transforman lo que algunos llaman la diversidad biocultural, pero que también extiende el Caribe a donde sus representantes reclaman esa comunidad imaginada de la que son parte, bien sea en el Atlántico Norte o el Cono Sur. Estos flujos, redes y conexiones que incrementan la heterogeneidad sociomaterial del Caribe es lo que hace visible este número especial sobre las migraciones en el Caribe, editado por Jorge Enrique ElíasCaro (ver Elías-Caro, 2018 en este número).

En la editorial del número previo a este, propuse que esa heterogeneidad cambiante que caracteriza el Caribe, podría indicar que nunca fuimos Caribe, pues no hay tal cosa pura y estable (algo que los antropólogos, por fortuna, ya entendimos y sabemos que aplica para todo). Pero curiosamente, Trouillot (2011), tal y como se cita en el epígrafe propone, también retomando a Latour (2007[1991]), que siempre hemos sido modernos, para hacer referencia al papel de espacios como el Caribe en la producción de aquello llamado modernidad, aunque al final plantee que "la lección más importante del caribe es, como yo la veo, una formidable: que la modernidad nunca fue, y nunca podría ser, lo que dice ser" (Trouillot, 2011: 95).

Al igual que Latour, otro autor de los estudios sociales de la ciencia y la tecnología nos puede ofrecer herramientas para entender lo Caribe, así, la expresión más que uno menos que muchos de Mol (2002) podría aplicarse al Caribe, nunca completo (fractal), siempre en proceso, geografía cambiante e interconectada con otras. Disimiles narrativas históricas que se cruzan: conquistas, 
comercios, esclavización, genocidios, migraciones, destierros, colonialismo, modernización y desarrollo; Identidades múltiples que se conectan parcialmente. Así lo Caribe no puede reducirse a una sola idea monolítica, la misma categoría es apropiada y resignificada de diferentes maneras por sus usuarios o consumidores; en tal sentido, es irrepresentable.

\section{REFERENCIAS BIBLIOGRÁFICAS}

Balanta, G. (2015). El abrazo por la vida y la libertad. Revista CS, (16), 243-246. Recuperado de: 10.18046/recs.i16.2045.

Camacho-Navarro, E. (2018). Imaginarios del progreso. Representaciones tecnológicas en el Circuncaribe mediante tarjetas postales (18991930). El caso de Costa Rica. Jangwa Pana, 17(3). Doi: 10.21676/16574923.2544

Guerrón-Montero, C. (2011), On Tourism and the Constructions of 'Paradise Islands' in Central America and the Caribbean. Bulletin of Latin American Research, 30(1): 21-34. Doi: 10.1111/j.1470-9856.2010.00453.x.

Herrera-Llamas, J.A., Alvarado-De Lima, J.A., Herrera-Aguilar, L. (2018). Globalización y pobreza: propulsores de las migraciones internacionales contemporáneas. Jangwa Pana, 17(3), Doi: 10.21676/16574923.2491

Jenson, D. (2010). The Writing of Disaster in Haiti: Signifying Cataclysm from Slave Revolution to Earthquake. En: Haiti Rising: Haitian History, Culture and the Earthquake (ed. Martin Munro). Liverpool. Liverpool University Press, pp. 102-112.

Martínez-Dueñas, W. (2016). Flujos y redes multinaturales: un recorrido por mundos no [solo] modernos en Puracé, Colombia. Popayán: Editorial Universidad del Cauca.

Martínez-Dueñas, W. y Arias-Ocampo, A. (2017). Jangwa Pana, el Caribe y "Publish or Perish". Jangwa Pana, 16(1) 9-26. Doi: 10.21676/16574923.1994.

Martínez-Dueñas, W. y Perafán, A. (2017). "Pensando la conservación desde el multinaturalismo en una localidad indígena de los andes colombianos". Universitas Humanística, 84, 77-107. Doi: doi:10.11144/Javeriana.uh84.pcml

Martínez-Dueñas W. y Perafán A. (2018). Postsostenibilidad: notas antropológicas para imaginar otros futuros comunes. Santa Marta, Colombia: Editorial Unimagdalena.

Mackenbach, W. (2011). ¿De la nación al toutmonde? Problemas, retos y perspectivas de los estudios regionales de Centroamérica y el Caribe. Pensamiento Actual, 11(16), 13-30.

Mol, A. (2002). The body multiple: Ontology in medical practice. Duke University Press.

Robles-Santana, M. A. (2018). Acercamiento a la histórica migración de mujeres indígenas Mamde Guatemala a las fincas cafetaleras de Chiapas (México). Análisis de su situación actual. Jangwa Pana, 17(3), Doi: 10.21676/16574923.2489.

Trouillot, M. R. (1992). The Caribbean region: an open frontier in anthropological theory. Annual Review of Anthropology, 21(1), 19-42.

Doi:10.1146/annurev.an.21.100192.000315.

Trouillot, M. R. (2011). Moderno de otro modo. Lecciones caribeñas desde el lugar del salvaje. Tabula Rasa, (14), 79-97. 